\title{
Evaluation of van der Waals density-functionals for layered materials
}

\author{
Sherif Abdulkader Tawfik and Michael J. Ford* \\ School of Mathematical and Physical Sciences, University of Technology Sydney, Ultimo, New South Wales 2007, Australi币 \\ Tim Gould

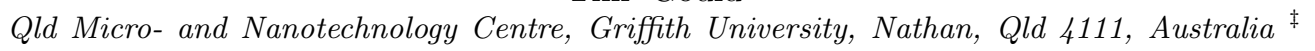 \\ Catherine Stampfl \\ School of Physics, The University of Sydney, New South Wales, 2006, Australia
}

\begin{abstract}
In 2012, Björkman et al. posed the question "Are we van der Waals ready?" [J. Phys.: Condens. Matter, 2012, 24, 424218] about the ability of ab initio modelling to reproduce van der Waals (vdW) dispersion forces in layered materials. The answer at that time was no, however. Here we report on a new generation of vdW dispersion models and show that one, fractionally-ionic atom (FIA) theory with many-body dispersions, offers close to quantitative predictions for layered structures. Furthermore, it does so from a qualitatively correct picture of dispersion forces. Other methods, such as D3 and optB88vdW also work well, albeit with some exceptions. We thus argue that we are nearly vdW ready, and that some modern dispersion methods are accurate enough to be used for nanomaterial prediction, albeit with some caution required.
\end{abstract}

\section{INTRODUCTION}

van der Waals (vdW) heterostructures [1, and nanoscience more generally, promise to transform science and technology by offering controllable material properties at the nanoscale. But many challenges must be met for the unprecedented benefits of heterostructures to be realised in technology. Not least of these is understanding what combinations of two-dimensional (2D) layers are both useful and structurally stable and, relatedly, how we can engineer structures to improve stability.

Significant work must thus be carried out to devise useful heterostructures, especially working out what combinations of layer types are useful and feasible. Isolating 2D layers is difficult, however. Assembling heterostructures is more difficult [1]. Thus, studying even a small representative space of interesting heterostructure seems like an impossible task for experimental laboratories.

Conveniently, heterostructure science has been paralleled by advances in computer modelling [2, 3, which offers the ability to scan large spaces of candidate materials quickly and efficiently. Prediction of heterostructure properties relies, at a minimum, on two major factors: an ability to reproduce lattice parameters, and thus basic geometries; and an ability to reproduce energies and their differences, and thus to understand the relative stability of different geometries. A good method must thus be able to reproduce these properties if it is to offer reliable results. Otherwise time can be wasted by experimentally exploring poor candidates misidentified as good by the virtual screening process. More worryingly, good candidates might never make it past the virtual screening process. Both hamper technological progress.

In this Letter we report an assessment of modern vdW dispersion approaches on a representative sample of $2 \mathrm{D}$ materials, including graphene, boron nitride,
$\mathrm{MoS}_{2}, \mathrm{MoSe}_{2}, \mathrm{MoTe}_{2}, \mathrm{WS}_{2}, \mathrm{PdTe}_{2}, \mathrm{TaS}_{2}, \mathrm{TaSe}_{2}, \mathrm{HfS}_{2}$, $\mathrm{HfSe}_{2}, \mathrm{HfTe}_{2}$. Our tests, building on previous work by Björkman and coworkers [4, 5] are designed to interrogate how well modern approaches can deal with the most basic properties of heterostructures. They thus include an important additional test not previously considered by Björkman at al: the quality of energy differences between different structural arrangements of homostructures. This test is critically important as it shows that a method not only works well in optimal homostructures, where it may benefit from a cancelattion of errors at the opimal interlayer spacing, but is also likely to work well in heterostructures which, due to the presence of incommensurate lattices, involve layered structures and their interactions in a range of relative configurations $[6]$.

We compare the predictive accuracy of 11 modern vdW methods against the predictions of the random-phase approximation (RPA) [7 9] which have been established as one of the most accurate methods for describing the physics of vdW materials [10, 11. Quantum MonteCarlo methods (QMC), widely considered to be highly accurate, have been applied for predicting the interlayer distance and the binding energy of graphite [12, 13]. QMC, however, has only been applied to a limited number of systems, while RPA has been applied for a wide range of systems. Our tests show that the fractional ionic atoms method [14] (referred to here as FIA for notational brevity - the method is more fully described as MBD@rsSCS/FI+ER as per the original paper) achieves a useful balance between the accurate prediction of the lattice constants, energies, and energy differences. 


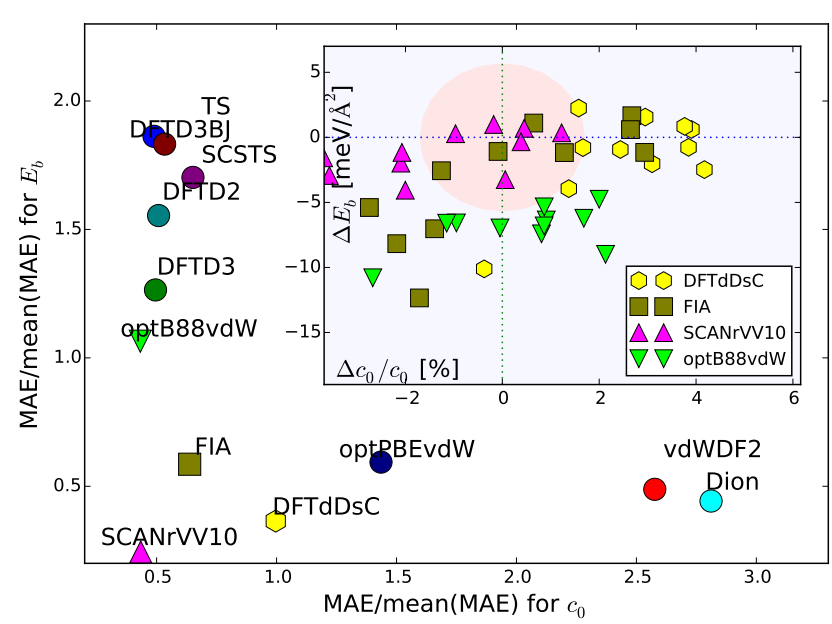

FIG. 1. (Main) Plot of the normalised mean average error (MAE) in the binding energy and lattice spacing for all tested methods. (Inset) Scatter plot of the four best methods showing all tested materials. The oval indicates the optimal goal of $\pm 2 \%$ for $c_{0}$ and $\pm 5 \mathrm{meV} / \mathrm{fu}$ for $E_{b}$.

\section{THEORY}

Dispersion forces, or van der Waals, forces, are weak forces that arise from the coupling between charge fluctuations in quantum systems. There has been a steady improvement in $a b$ initio methods which account for a full description of chemical and dispersive forces. Broadly speaking, these fall into three categories: semi-empirical (SE) "DX" models from Grimme et al. [15]17, models based on atomic polarizabilities (AP-D) modified by the electron density [14, 18, 20], and full density functional approximations (DFA) based on pairwise dispersion models using only the density [21, 22]. Table I] summarises the methods applied in this Letter, categorized according to the above scheme. Further details of all methods are provided in the original works, and more detailed summaries of modern dispersion approaches are provided in Refs. 22, 23]. Here we focus on methods which correct generalized gradient approximations, as these are the most widely available and easily employed class of density functionals.

To understand the qualitative advantages and disadvantages of dispersion methods, we must first focus on the competing pictures of dispersion forces: the first, more dominant amongst chemists, is that interactions between atoms can be modified and then summed to get the total interaction. The second, more dominant amongst physicists, employs polarizability models from Lifshitz which are based around the physics of macroscopic solids.

Dobson recently described how these two pictures can be connected to one another 10 and thus applied to nanostructures which share properties with molecules and bulk solids. He divided contributions to dispersion
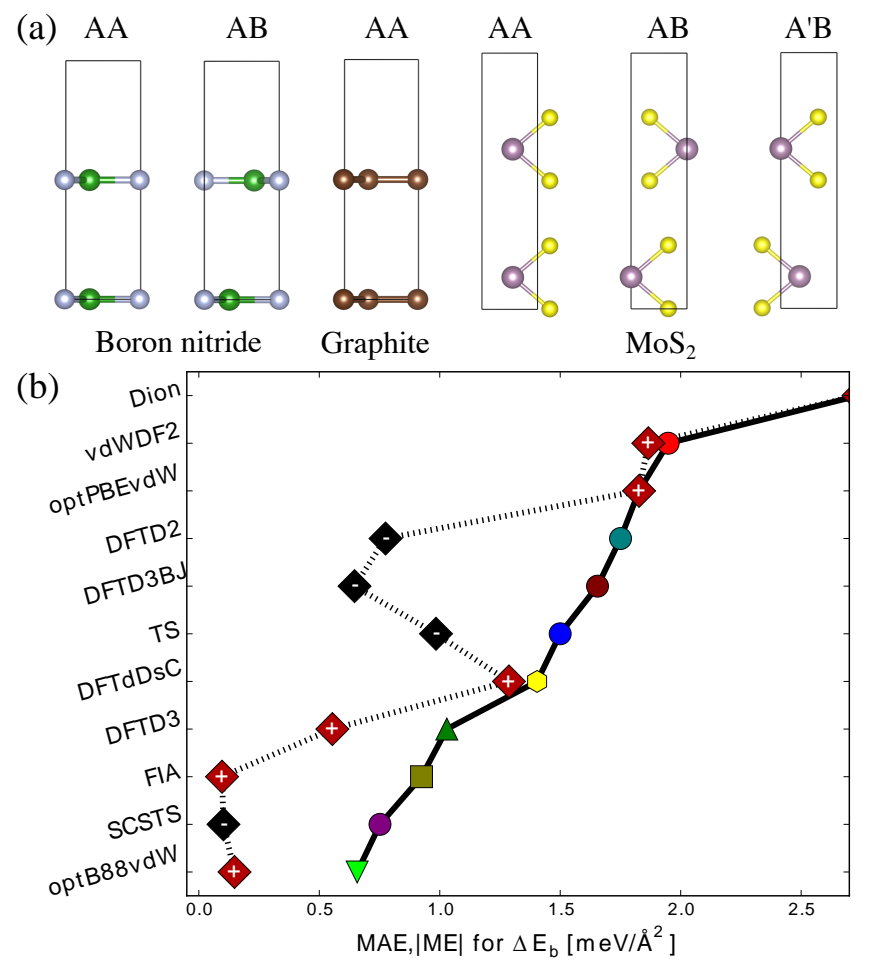

FIG. 2. (a) The side-view of the various stacking orders for graphene, hBN and $\mathrm{MoS}_{2}$. (b) Plot of the MAE (thick line and markers) and ME (dashed line, with indicators of sign) for energetic differences $\Delta E=E_{0}^{G 1}-E_{0}^{G 2}$ that serve as a proxy quality metric for heterostructures. Here $G 1$ and $G 2$ indicate different stackings of the same material.

forces into three types of "non-additivity" effects. His first, here called "Dobson-A" effects, involves contributions from chemical environments and is present in all useful theories of dispersion forces. His second, Dobson$\mathrm{B}$ effects, involves many-body interactions known from Lifshitz theory and is present in the RPA, and the Many-body dispersion (MBD) [19] class of approximations which has been used to show their vital importance for describing nanostructure binding [24 26]. His third, Dobson-C, involves metallic/insulating physics and is approximated in MBD [24] and fully present in RPA. Dobson-C effects mostly affect asymptotic physics [2730] [that is, the energetic behavior of layered materials for large $(\gg 10 \mathrm{~nm}$ ) separation distances between the layers] and are unlikely to be relevant to typical studies of two-dimensional heterostructures.

In the computational assessment of the quality of the $\mathrm{vdW}$ methods, a critical concern is the identification of benchmarks. In such studies, the two primary quantities that are predicted are the $c_{0}$ lattice parameter and the layer binding energy, $E_{b}$, and these must be tested against suitable benchmark data. Lattice parameters are known accurately from experiments, which can serve as a benchmark, at least up to contributions from the zero- 
TABLE I. The computational methods used in this study, the key reference of each method, the classification of the method [whether based on the semi-empirical methods of Grimme (SE), atomic polarizabilities modifed by the density (AP-D) or pure functionals of the electronic density (DFA), which Doboson nonadditivity types [10] are supported [? means partially supported]. The final columns " $c_{0}$ " and " $E_{b}$ group the methods by their success in predicting $c_{0}$ and/or $E_{b}$. The FIA method is the one that is closest to the accuracy of the RPA method.

\begin{tabular}{|c|c|c|c|c|c|c|c|}
\hline \multirow[t]{2}{*}{ Method } & \multirow[t]{2}{*}{ Ref. } & \multirow[t]{2}{*}{ Class } & \multicolumn{3}{|c|}{ Non-additivity } & \multicolumn{2}{|c|}{ Quality } \\
\hline & & & & $\mathrm{B}$ ? & $\mathrm{C} ?$ & $c_{\mathrm{C}}$ & $E_{b}$ \\
\hline RPA & 31 & ACFD & $\checkmark$ & $\checkmark$ & $\checkmark$ & & \\
\hline SCAN-rVV10 & 32 & DFA & $\checkmark$ & $x$ & $x$ & $\checkmark$ & $\checkmark$ \\
\hline FIA & 14 & AP-D & $\checkmark$ & $\checkmark$ & $?$ & & \\
\hline $\mathrm{TS}$ & 18 & AP-D & $\checkmark$ & $x$ & $x$ & & \\
\hline DFTD3BJ & 17 & S-E & $\checkmark$ & $?$ & $x$ & & \\
\hline SCSTS & 19 & AP-D & $\checkmark$ & $\checkmark$ & $\times$ & & \\
\hline DFTD2 & 15 & S-E & $\checkmark$ & $x$ & $x$ & & $\times$ \\
\hline DFTD3 & 16 & S-E & $\checkmark$ & $?$ & $x$ & & \\
\hline optB88vdW & 21 & DFA & $\checkmark$ & $x$ & $x$ & & \\
\hline DFTdDsC & 20 & AP-D & $\checkmark$ & $\bar{x}$ & $\bar{x}$ & & \\
\hline optPBEvdW & 21 & DFA & $\checkmark$ & $x$ & $x$ & & \\
\hline vdWDF2 & 33 & DFA & $\checkmark$ & $x$ & $\times$ & $x$ & \\
\hline Dion & 22 & DFA & $\checkmark$ & $x$ & $x$ & & \\
\hline
\end{tabular}

point energy. The situation for binding energies $E_{b}$ is rather more challenging, however, given the inaccuracies encountered in the indirect measurement of small energy differences. Therefore, for $E_{b}$, we use instead published RPA values for $E_{b}$, 4, 6, 28, 34, as benchmarks for the present study, as done in previous studies [4. Note that the RPA gives good agreement with experiment for lattice constants [4, 35] and includes all of Dobson's nonadditivity classes A, B and C. It is thus likely to carry a complete picture of binding in layered materials.

With the qualitative picture, and quantitative benchmarks established, we can now consider the models in our study. All three categories (SE, AP-D and DFA) of dispersion methods have seen steady improvements in accuracy over the past decade [5]. Table I reports the list of approaches tested here, representing recent iterations in each category (we note that MBD@rsSCS has problems in transition metal dichalcogenides [14] and was thus excluded from our studies).

Any new method is assessed by performing statistical tests on the outcomes of calculations for a set of benchmark systems, compared against higher-level theory (or experimental) data. Usually, such benchmarking has lacked the inclusion of $2 \mathrm{D}$ heterostructures, and has been based only on atomic and molecular systems. Tests are typically reported only for graphite and hexagonal boron nitride, if anything. Methods may thus suffer from inaccuracies in predicting the properties of the interaction in $2 \mathrm{D}$ materials generally. Here, we seek to remedy this deficiency to understand which methods are suitable for calculations of $2 \mathrm{D}$ and related systems.

At the pairwise level, we test Grimme's D2 empirical correction [15] (DFTD2), Grimme's D3 empirical correction [16] in its original form (DFTD3) and with BeckeJohnson damping (DFTD3BJ), the exchange-hole based correction of Steinmann and Corminboeuf 20. (DFT$\mathrm{dDsC}$ ), and the Tkatchenko-Scheffler method [18 (TS) method, and its self-consistent screened version [19] (SCSTS). The many-body dispersion method (MBD@rsSCS) [19], based on SCSTS but with explicit many-body Dobson-B contributions collapses in calculations of materials with large polarizabilities, including transition metals in the fourth and fifth rows of the periodic table. We therefore instead use a recently introduced modification of the MBD@rsSCS method, the FIA method, which involves a more sophisticated treatment of polarizabilities by drawing from the properties of fractional ions [36]. FIA has been shown to perform as well as MBD in molecular tests, but significantly outperforms it in strongly polarizable systems, such as transition metal dichalcogenides, interactions involving ions, and benzene dimers [14, 37, 38. The other computational methods are all based around the two-point vdW density-functional approach of Dion et al. 22. This vdW correction is applied with the revPBE density functional [21, the optPBE density functional 21 (optPBEvdW) and the optB88 density functional 21] (optB88vdW). Also, in a form modified by Lee et al., it is combined [33] with the BP86 density functional [39] (vdWDF2).

All calculations are performed using VASP 5.4.1 [40], where the valence electrons are separated from the core by use of projector-augmented wave pseudopotentials (PAW) [11. The energy cut-off for the plane-wave basis functions was set at $500 \mathrm{eV}$. The energy tolerance for the electronic structure determinations was set at $10^{-7}$ $\mathrm{eV}$ to ensure accuracy. The diversity of the structures investigated here ensure that our results are not dependent upon the choice of systems. We use $\mathbf{k}$-space grids of $9 \times 9 \times 3$ for graphene and boron nitride, $11 \times 11 \times 3$ for $\mathrm{PdTe}_{2}$, and $15 \times 15 \times 3$ for the rest, based on energy convergence. Geometry optimizations were performed for all structures, terminating when the forces on all atoms fell below $0.01 \mathrm{eV} / \AA$. MBD and FI are calculated using the reciprocal space implementation [42. The inplane lattice parameters $a$ are kept fixed at the respective experimental values, in accordance with previous work [4. Small differences between the experimental and the equilibrium theoretical $a$ lattice parameter do not significantly affect the results for equilibrium $c_{0}$ and $E_{b}$.

\section{RESULTS}

Our calculations of $c_{0}$ and $E_{b}$ naturally divide the vdW methods considered into two groups, as 
summarized in Table II one group tends to perform poorly for $c_{0}$ and acceptably for $E_{b}$. The other does the opposite. In order to quantify this classification, we display in Fig. 1 the normalized mean average error values of $c_{0}$ or $E_{b}$, given by $N_{c_{0} / E_{b}}(v d W)=$ $M A E_{c_{0} / E_{b}}(v d W) /\left[\frac{1}{N_{v d W}} \sum_{v d W} M A E_{c_{0} / E_{b}}(v d W)\right]$,

where $v d W$ labels the different $N_{\mathrm{vdW}}=12(11$ tested here and SCAN+rVV10) methods considered, and $M A E_{c_{0} / E_{b}}$ is the mean average error across the 11 layered compounds. For each of the 11 vdW methods tested here (plus results for SCAN+rVV10 from the literature[32]), the MAE for the prediction of $c_{0} / E_{b}$ is given by $M A E(v d W)=\sum_{i}^{11}\left|X_{R P A}-X_{v d W}\right| / 11$, where $i$ iterates over the number of structures, $X_{R P A}$ is the benchmark $c_{0}$ or $E_{b}$ value, and $X_{v d W}$ is the calculated $c_{0}$ or $E_{b}$ value. According to this scheme, an accurate vdW method is one whose $N_{c_{0}}(v d W)$ and $N_{E_{b}}(v d W)$ are both signficantly less than one. That is, such a method is able to closely reproduce the results obtained using RPA.

The grouping discussed above is immediately obvious from this plot. Results clearly fall into two groups of vdW methods: group I that includes TS, DFTD3BJ, SCSTS, DFTD2, DFTD3, optB88vdW, and group II that includes DFTdDsC, optPBEvdW, vdWDF2 and Dion. While each group has tradeoffs, an important feature made clear by this figure is that the FIA methods sits at the intersection of the two, and thus achieves a balance between energies and lattice constants. Note that standard MBD theory fails completely for transition metal dichalcogenides [14] and has been left out of these tests.

We can identify the four best methods from Fig. 1 FIA, DFTdDsC, DFTD3 and optB88vdW, based on the observation that they have the smallest $N_{c_{0} / E_{b}}(v d W)$ values. For these four methods, and for the $11 \mathrm{vdW}$ materials considered, the inset displays a scatter plot of the values of $\Delta c_{0} / c_{0}$ and $\Delta E_{b}$. We identify a region (in pink) that marks the optimal accuracy targets for each of the four methods across the set of structures. The values $\pm 2 \%$ [32] and $\pm 5 \mathrm{meV} / \mathrm{fu}$ (functional unit) for $\Delta c_{0} / c_{0}$ and $\Delta E_{b}$, respectively, are chosen based on the following criteria: for the $c$ lattice parameter, it is on the scale of zero-point energy effects; for $\Delta E_{b}$, it is about what one expects for a typical "registry" difference (e.g., between $\mathrm{AA}^{\prime}$ ' and $\mathrm{AB}$ in $\mathrm{MoS}_{2}$ - discussed in more detail below). The inset in the figure shows that, while no method achieves the desired performance across even most materials, the FIA method is the one that has the most results within the shaded circle.

So far we have focused on properties of optimal homostructures, i.e. layered materials in which the layers have been arranged in their lowest energy configuration. In future, the primary goal of studying layered materials is likely to shift to heterostructures, in which perfect registry is impossible to achieve due to different lattice parameters. Thus, it is important to ask whether or not methods are sufficiently accurate for heterostructures. Reliable heterostructure benchmarks from RPA or other high-level theories are beyond current computational limits, however, making benchmarking impossible. Graphene/BN being a notable exception. 6]

To overcome this limitation, we test instead an important and related property of homostructures, namely the difference in energies between structures arranged in non-optimal stackings [illustrated in Fig 2(a)], and the energy of the lowest energy state. Since heterostructures involve many atoms outside optimal registry, the ability of a method to reproduce these energy differences will be important for accurate calculations. Furthermore, these energies involve an interplay between dispersive and electrostatic forces 43, and thus methods cannot rely on any convenient cancellation of errors near the optimal lattice point and must reproduce both with sufficient accuracy. Thus, although imperfect, these tests are likely to be the best available proxy for heterostructure physics.

We examine the influence of stacking on the contribution of each of the 11 methods, and display the results in Fig. 2(b). We focus on the following stacking configurations for graphene, $\mathrm{hBN}$ and $\mathrm{MoS}_{2}$ : $A A$ in graphene, hBN and $\mathrm{MoS}_{2}, A B$ in $\mathrm{hBN}$ and $\mathrm{MoS}_{2}$ and $A$ ' $B$ in $\mathrm{MoS}_{2}$. These cases all have reliable RPA benchmark data 6, 34, and include both small and large energy differences to cover different physical regimes that may be encountered in real heterostructures. Our results show that two of the four methods already identified as good, namely optB88vdW, and FIA, are also the best at capturing energy differences (note, SCSTS does best on the energy difference tests, but is worst for binding energies). The consistent behavior of the four methods emphasizes their general accuracy in various situations.

Finally, in our assessment of the various dispersion approaches, we devote some special attention to $\mathrm{PdTe}_{2}$, which exhibits the interesting property that the covalent and vdW dispersion forces compete; according to our calculations, the application of PBE (without any vdW dispersion model) yields a $c_{0}$ of $5.327 \AA$, and a $E_{b}$ of 18.2 meV, which are much closer to the experimental values than the corresponding quantities for other TMC compounds when calculated using PBE. The performance of the $\mathrm{vdW}$ dispersion methods in the case of this compound is important in identifying the behavior of the dispersion forces in an extreme case. Out of the $11 \mathrm{vdW}$ methods, the ones that have lowest errors in $E_{b}$ are DFTdDsC, SCSTS, TS and optPBEvdW, while the ones with the lowest errors in $c_{0}$ are DFTD2, DFTD3, DFTD3BJ, DFTdDsC, FIA, SCSTS, TS and optB88vdW. This means that, while the FIA method, on average achieves the tradeoff, other methods can be more accurate in selected cases. 
Having established that the FIA method has the most competitive agreement with RPA among the GGA-based methods investigated in the present Letter, we note that recently published results on the dispersion-corrected meta-GGA SCAN+rVV10 32 show it gives a superior performance even to FIA, with about $40 \%$ average improvements to lattice constants and energies (no results are available for stacking energies), despite poor performance [5] for PBE+rVV10 in the same systems. However, a critical issue with SCAN+rVV10 is the computational performance: given the complexity of the evaluation of the kinetic energy density in meta-GGA based methods, would SCAN+rVV10 suffer from higher computational complexity? To quantify this, we have performed a full-relaxation calculation on a hybrid bilayer system composed of graphene and WS2. This system has $3 \times 3$ WS2 and $4 \times 4$ graphene, and both calculations started with the same initial atomic structure and with an energy cut-off of $600 \mathrm{eV}$ (which is higher than the value used by rest of the calculations here, because this is required for the convergence of the meta-GGA functional). The full relaxation of this system using SCAN+rVV10 on 64 cores required $\sim 4.1$ times the time FIA requires to perform the same calculation.

Therefore, while SCAN+rVV10 and FIA have competitive accuracies, especially when compared to other methods tested here, an FIA calculation takes far less time than $\mathrm{SCAN}+\mathrm{rVV} 10$. We point out that the recent re-parametrization of the $\mathrm{PBE}+\mathrm{rVV10}$ method, known as PBE+rVV10L [44] has been reported to yield reasonably accurate results that are comparable to SCAN+rVV10. But this method requires the tuning of a fitting parameter for different systems which makes it of limited applicability in general since one cannot us the same method to treat, e.g., a molecule adsorbed to a layered surface. A similar strategy was previously employed by Björkman et al [4] who simply scaled VV10 energies by $66 \%$ to better match RPA results for layered materials.

\section{CONCLUSIONS}

We have investigated the accuracy of 11 vdW dispersion methods for the prediction of the geometric and energetic properties of 11 representative vdW materials, and we have found that there is a tradeoff between the accuracy in determining the geometric and energetic properties. Out of the 11 methods, we report that the recently introduced FIA methods achieves the tradeoff, and that the FIA, DFTD3, optB88vdW and DFTdDsC methods achieve high accuracy with respect to the other methods. Two methods out of these four, namely FIA and optB88vdW, deliver more accurate predictions compared to the other two for $2 \mathrm{D}$ materials with nonequilib- rium stacking orders.

We believe that the ability of most methods to get good lattice parameter or energies, but not both, points to underlying problems in the abiliy of their polarizability models to adjust to different geometries (Dobson-A and -B non-additivity), especially when the layers are brought close to contact. Consequently, the damping function which connects the dispersion correction to the underlying exchange-correlation functional is unable to meet the competing demands of getting both energies and lattice parameters right. Only methods with very good underlying polarizability physics, such as FIA or SCAN+rVV10 45, give sufficiently good dispersion energies near contact to reproduce geometries and energies together. This argument is supported by out-ofequilibrium results for the benzene dimer 38 .

It is interesting to note that each of the four best methods found here represents the latest generation of a different class of vdW method, highlighting the steady improvements in each class. Our work thus suggests two important elements for the success of future vdW methods: (a) achievement of the tradeoff between geometry and energy characteristics, and (b) inclusion of the physical principles that drive the current methods, perhaps by borrowing "best practice" from methods of a different class.

Importantly, our work highlights the need to test and develop methods using a wide range of systems. Most dispersion methods are optimized and initially tested on small molecular systems, due in part to the availablility of high-quality benchmark data. However, as we have shown here this does not necessarily mean they work well in layered systems. Nor, presumably, when molecules are physi- or cheimsorpbed onto surfaces. We feel this motivates the need for better benchmark data of difficult systems.

Let us finally draw our attention to the most promising route for improving dispersion force modelling. Here we focused on generalized gradient approximation (GGA) based approaches, due to their wide availability. The meta-GGA SCAN+rVV10 offers superior performance to any of the GGA-based approaches, however, despite known problems with PBE+VV10 for layered systems and a complete absence of Dobson-B contributions. This suggests that (modified) meta-GGAs may offer a superior starting point for dispersion corrections. Combining the most reliable dispersion corrections here (e.g. FIA) with meta-GGAs may thus offer the possibility of even better performance going into the future. Progress along these lines is being pursued

This research was funded by the Australian Government through the Australian Research Council (ARC DP160101301). Theoretical calculations were undertaken with resources provided by the National Computational Infrastructure (NCI) supported by the Australian Government and by the Pawsey Supercomputing Centre 
funded by the Australian Government and the Government of Western Australia.

* mike.ford@uts.edu.au

† sherif.abbas@uts.edu.au

¥ t.gould@griffith.edu.au

[1] A. Geim and I. Grigorieva, Nature 499, 419 (2013).

[2] T. Gould, S. Lebègue, T. Björkman, and J. Dobson, in $2 D$ Materials, edited by J. J. B. Francesca Iacopi and C. Jagadish (Elsevier, 2016), vol. 95 of Semiconductors and Semimetals, pp. 1-33, URL http://www.sciencedirect.com/science/article/ pii/S0080878416300035

[3] J. Paul, A. K. Singh, Z. Dong, H. Zhuang, B. C. Revard, B. Rijal, M. Ashton, A. Linscheid, M. N. Blonsky, D. Gluhovic, et al., Journal of Physics: Condensed Matter (2017).

[4] T. Björkman, A. Gulans, A. V. Krasheninnikov, and R. M. Nieminen, Phys. Rev. Lett. 108, 235502 (2012).

[5] T. Björkman, A. Gulans, A. V. Krasheninnikov, and R. M. Nieminen, J. Phys.: Condens. Matter 24, 424218 (2012), URL http://stacks . iop.org/0953-8984/24/i= $42 / \mathrm{a}=424218$.

[6] N. Leconte, J. Jung, S. Lebègue, and T. Gould, Phys. Rev. B 96, 195431 (2017), URL https ://link .aps .org/ doi/10.1103/PhysRevB.96.195431

[7] H. Eshuis, J. E. Bates, and F. Furche, Theor. Chem. Acc. 131, 1 (2012).

[8] J. F. Dobson and T. Gould, J. Phys.: Condens. Matter 24, 073201 (2012).

[9] X. Ren, P. Rinke, C. Joas, and M. Scheffler, J. Mater. Sci. 47, 7447 (2012).

[10] J. F. Dobson, Int. J. Quantum Chem. 114, 1157 (2014).

[11] B. Sachs, T. O. Wehling, M. I. Katsnelson, and A. I. Lichtenstein, Phys. Rev. B 84, 195414 (2011), URL https://link.aps.org/doi/10.1103/PhysRevB. 84.195414

[12] L. Spanu, S. Sorella, and G. Galli, Physical review letters 103, 196401 (2009).

[13] P. Ganesh, J. Kim, C. Park, M. Yoon, F. A. Reboredo, and P. R. C. Kent, Journal of Chemical Theory and Computation 10, 5318 (2014), pMID: 26583215, https://doi.org/10.1021/ct500617z, URL https://doi. org/10.1021/ct500617z

[14] T. Gould, S. Lebègue, J. G. Ángyán, and T. Bučko, J. Chem. Theor. Comput. 12, 5920 (2016).

[15] S. Grimme, J. Comp. Chem. 27, 1787 (2006).

[16] S. Grimme, J. Antony, S. Ehrlich, and H. Krieg, The Journal of Chemical Physics 132, 154104 (2010), URL http://link.aip.org/link/?JCP/132/154104/1

[17] S. Grimme, S. Ehrlich, and L. Goerigk, Journal of computational chemistry 32, 1456 (2011).

[18] A. Tkatchenko and M. Scheffler, Phys. Rev. Lett. 102, 073005 (2009-02).

[19] A. Tkatchenko, R. A. DiStasio, R. Car, and M. Scheffler, Phys. Rev. Lett. 108, 236402 (2012-06).

[20] S. N. Steinmann and C. Corminboeuf, The Journal of chemical physics 134, 044117 (2011).

[21] J. Klimeš, D. R. Bowler, and A. Michaelides, Journal of
Physics: Condensed Matter 22, 022201 (2009).

[22] M. Dion, H. Rydberg, E. Schröder, D. C. Langreth, and B. I. Lundqvist, Physical review letters 92, 246401 (2004).

[23] J. Hermann, R. A. DiStasio, and A. Tkatchenko, Chem. Rev. 117, 4714 (2017), pMID: 28272886.

[24] R. A. DiStasio, O. A. von Lilienfeld, and A. Tkatchenko, Proc. Natl. Acad. Sci. U.S.A. 109, 14791 (2012), URL http://www.pnas.org/content/109/37/14791. abstract.

[25] V. V. Gobre and A. Tkatchenko, Nat Commun 4, 2341 (2013).

[26] A. Ambrosetti, N. Ferri, R. A. DiStasio, and A. Tkatchenko, Science 351, 1171 (2016), ISSN 00368075, URL http://science.sciencemag.org/content/ 351/6278/1171.

[27] J. F. Dobson, A. White, and A. Rubio, Phys. Rev. Lett. 96, 073201 (2006).

[28] S. Lebègue, J. Harl, T. Gould, J. G. Ángyán, G. Kresse, and J. F. Dobson, Phys. Rev. Lett. 105, 196401 (2010).

[29] J. F. Dobson, T. Gould, and G. Vignale, Phys. Rev. X 4, 021040 (2014-05).

[30] J. F. Dobson, T. Gould, and S. Lebègue, Phys. Rev. B 93, 165436 (2016).

[31] J. Harl, L. Schimka, and G. Kresse, Phys. Rev. B 81, 115126 (2010), URL https://link.aps.org/doi/ 10.1103/PhysRevB.81.115126

[32] H. Peng, Z.-H. Yang, J. P. Perdew, and J. Sun, Physical Review X 6, 041005 (2016).

[33] K. Lee, É. D. Murray, L. Kong, B. I. Lundqvist, and D. C. Langreth, Physical Review B 82, 081101 (2010).

[34] J. He, K. Hummer, and C. Franchini, Phys. Rev. B 89, 075409 (2014)

[35] J. Harl and G. Kresse, Phys. Rev. Lett. 103, 056401 (2009).

[36] T. Gould and T. Bučko, J. Chem. Theory Comput. 12, 3603 (2016).

[37] J. Claudot, W. J. Kim, A. Dixit, H. Kim, T. Gould, D. Rocca, and S. Lebègue, The Journal of Chemical Physics 148, 064112 (2018).

[38] T. Gould, E. R. Johnson, and S. A. Tawfik (2018), URL https://chemrxiv.org/articles/Are_Dispersion_ Corrections_Accurate_Outside_Equilibrium_A_Case_ Study_on_Benzene/5917114

[39] J. P. Perdew, Physical Review B 33, 8822 (1986).

[40] G. Kresse and J. Furthmuller, Phys. Rev. B 54, 11169 (1996).

[41] P. E. Blochl, Phys. Rev. B 50, 17953 (1994).

[42] T. Bučko, S. Lebègue, T. Gould, and J. G. Ángyán, J. Phys.: Condens. Matter 28, 045201 (2016), URL http: //stacks.iop.org/0953-8984/28/i=4/a=045201.

[43] G. Constantinescu, A. Kuc, and T. Heine, Physical review letters 111, 036104 (2013).

[44] H. Peng and J. P. Perdew, Phys. Rev. B 95, 081105 (2017), URL https://link.aps.org/doi/10. 1103/PhysRevB.95.081105.

[45] Note1, note, the SCAN functional contains good shortrange dispersion contributions, but neglects long-range terms. It can therefore make up for deficiencies in rVV10, which are heavily damped near contact by the large damping parameter $b=15.7$. 